\title{
Renward Cysat und Paracelsus
}

\author{
Von Willem F. Daems*
}

Am 24. September 1541 starb Paracelsus in Salzburg. Sein geistiges Erbe traten diejenigen Freunde seiner Welt- und Menschenanschauung an, die man Paracelsisten nennt. Männer wie Michael Toxites (=Schütz, 1515-1581), Adam von Bodenstein (1528-1577), Leonhard Thurneisser (1530-1596), Petrus Severinus (1542-1602), Joseph Quercetanus (= Duchesne, ca.1544-1609), Gerhard Dorn (fl.1566-1584) und Theodore Turquet de Mayerne (1573-1655). Es gab aber auch - selbstverständlich muß man sagen-Gegner, bereits zu Paracelsus' Lebzeiten und besonders danach, Anti-Paracelsisten oder Galenisten wie der fanatische Basler Thomas Erastus (= Liebler, 1524-1583), der Paracelsus als «Bestie» und «grunzendes Schwein» einschätzte. Und dann gab es die toleranten Versöhner zwischen Galen und Paracelsus, wie Theodor Zwinger (1533-1588) und dessen Schwager Hans Jakob Wecker (1528-1584).

Von Renward Cysat (1545-1614), der vier Jahre nach Paracelsus' Tod sein Erdenleben begann, mag man sich also mit Recht fragen: Wie stand dieser zu Paracelsus? In den bis heute herausgegebenen Teilen der Collectanea zitiert Cysat den Hohenheimer zweimal, und dann legt er noch - in einem einseitigen Beitrag - Zeugnis seiner Bewunderung für ihn ab. Auch ist es bekannt, daß Cysat in seiner Bücherei Werke von Paracelsus besaß. Man lese Cysats «Würdigung» - diese Charakterisierung stammt von Josef Schmid ${ }^{1}$ des Paracelsus:

De Theophrasto Paracelso Magno

Diser ist ein hochvernampter medicus gsin siner zytt vngefarlich Anno Christi 1500 von dem adelichen stammen der Bombasten $v ß$ Schwaben geboren, vnd zuo Einsidlen jm Schwytzerland erzogen. Diser hatt ein nüwe form vnd gebruch jn der medicin erfunden durch sonderbare distillationes vnd subtiliteten jn kleiner oder ringer quantitet vnd andre seltzame vnd zuovor vnerhörte cabalistische heimlicheiten; derwegen ein großer theil jnne für einen nicromaticum vnd zauberer gehallten, besonder die Galenisten, deren kunst, procedur vnd form ze artznen er strax das widerspil gebrucht. Hatt zwar sine sachen ans liecht kommen laßen, wie dann syne büecher getruckt worden aber die recept vnd descriptiones so dunckel vnd mit frömbden namen, die man in rerum natura nit finden können. Hatt ouch ettliche sachen offenlich gnuog beschriben, die aber nit one grosses

* Kurze Mitteilung an der 25.(Jubiläums-) Generalversammlung der Schweizerischen Gesellschaft für Geschichte der Pharmazie, Luzern, 5./6. Juni 1982. 
abschühen erbarer vnd züchtiger oren, ouch nit geringer ergernuß gehört, ja nit one höchste schand vnd große todsünd verricht werden könntend. Alls zum exempel vnder anderm wie ein kind könnte gezyglet vnd geboren werden one ein wyb vnd vsserhalb wyplichs lybs etc.

Es habends jme ettliche alls discipuli wöllen nachthuon, sind aber angloffen.

Vnd jst noch hütt by tag ein hefftiger, großer stryt zwüschen den Paracelsisten vnd Galenisten; vervolgend ouch einandern vff $\mathrm{dz}$ v̈sserst.

Diser Paracelsus jst arbeitsam, ouch ein wunderbarlicher, erfarner Alchimist gsin, vnd doch jn armuott gstorben.

Er würdt von Valentino de Retijs hoch gerüempt, quod fuerit insignis philosophus stoicus et conscripserit perplurima opera in lucem edita, scilicet libros

\begin{tabular}{|c|c|c|c|}
\hline in philosophia & No 230 & in mathematica & No \\
\hline jn medicina & No 46 & de Theophrastia & No \\
\hline de republica & No 12 & de occultioribus et abstrusis & No 60 \\
\hline
\end{tabular}

Es hand aber sine büecher vil jrrthumben vnd vanitates, wölche von andern fürnemmen autoribus verworffen werdent.

Doch so jst es ye ein wunderbarliche gab gsin von einem solchen ingenio vnd ein miraculum naturae.

Josef Schmid, der Bearbeiter und Herausgeber der Collectanea chronica sagt dazu 2: « Er [Cysat] nennt Paracelsus «Magnus», den Großen.» Die Sachlage ist ein wenig anders, denn für seine «Würdigung» hat Cysat - so zeigt die Untersuchung - Gebrauch gemacht vom Brief «Ad lectorem» des Valentius de Retijs. Dieser nennt Paracelsus den Magnus! Wir lassen deshalb auch diesen Brief in der Toxites-Übersetzung ${ }^{3}$ von 1574 folgen:

«Valentinus de Rhetijs, von dem Theophrasto und seinen Schriften. Zum Leser. Philippus Theophrastus von Hohenheim, zu Einsideln im Schweitzerland geboren, ist von den Atheniensibus der große Paracelsus genant worden. da er hat 230 bücher in der Philosophei geschriben, 40 bücher in der artzenei, 12 de republica, 7 in Mathematica oder Astronomei, 66 von verborgnen und heimlichen künsten. Mehr hat er drei opera in ein buch gesetzt, welches er Theophrastiam genennt: Das erst heißt Archidoxa, in dem er lehret das rein vom unreinen zu scheiden: das ander Parasarchum, in welchem er de summo bono in aeternitate tractirt: das dritt Carboantes, in dem er die transmutationes in forma et esse beschreibet. Der Gellius Zemeus, hat von disem Teutschen Theophrasto philosopho, zum Passephallo Ceveo mit den worten geschriben: Im Teutschland ist jetzt ein junger mann, der gleichen in der ganzen welt nicht gefunden wird, der so fürtrefflich und köstlich in der Philosophei, Artznei, Astronomei, und vom Gemeinen nutz und dem rechten geschriben hat, das ich nicht anders glaub, er habs entweder aus einer wunderbarlichen angebornen influenz, oder aus unaussprechlicher gnad des heiligen geists, oder aber aus der bösen geister eingebung. dan was er nur für gering und schlecht haltet, das ist keinem menschen wol möglich zu erfaren und zu ergründen. ich kan mich nicht erinnern, das ich eines 


\section{DeTheophnaslo Graracelso Magno 1721628}

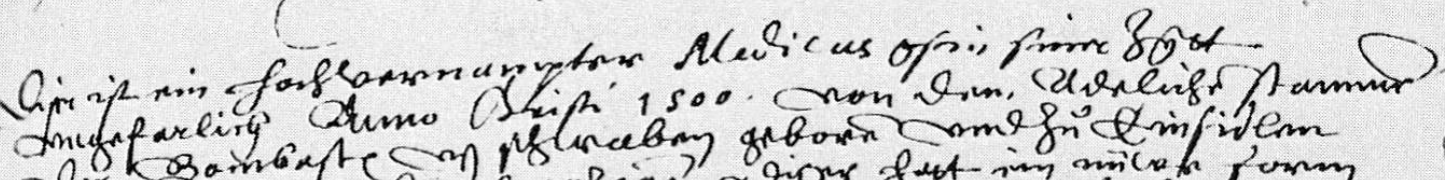

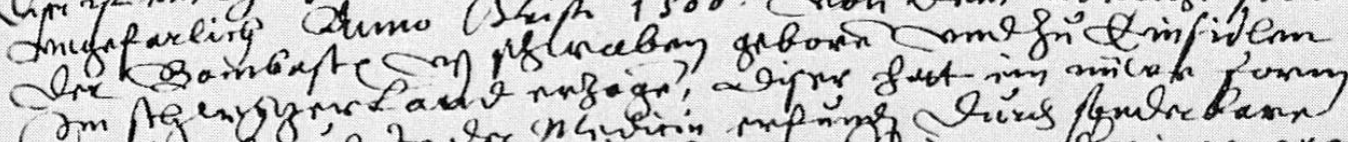

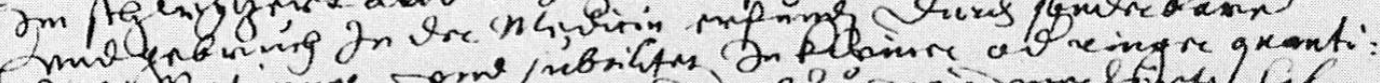

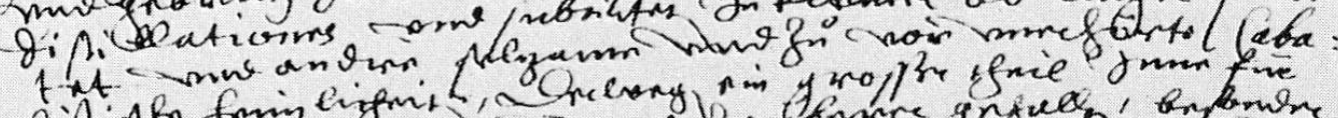
eiftiff frimengmi

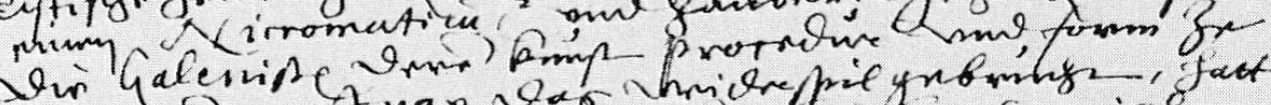

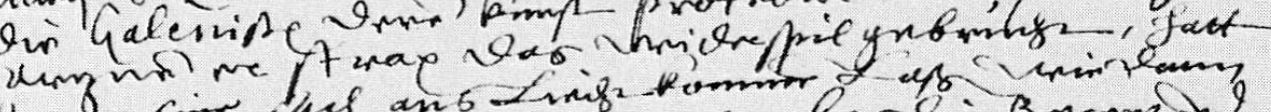

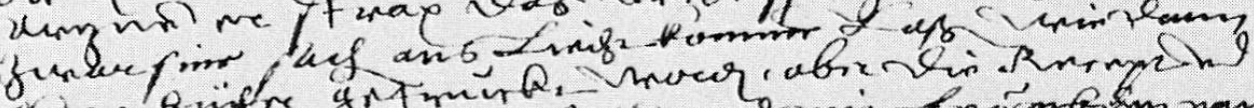

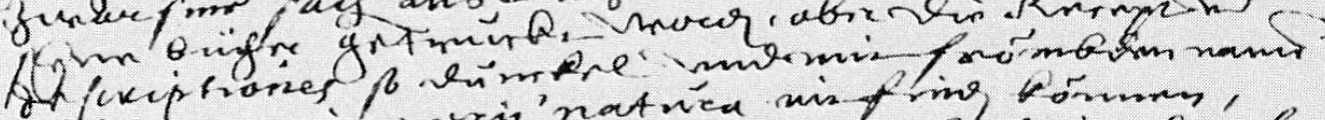

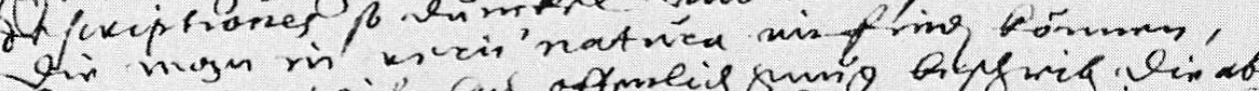

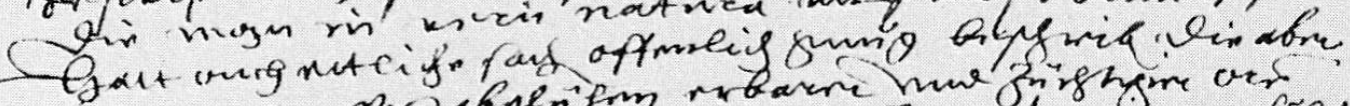

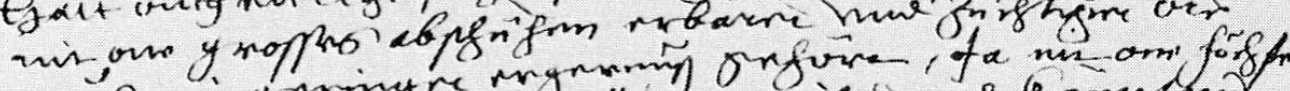

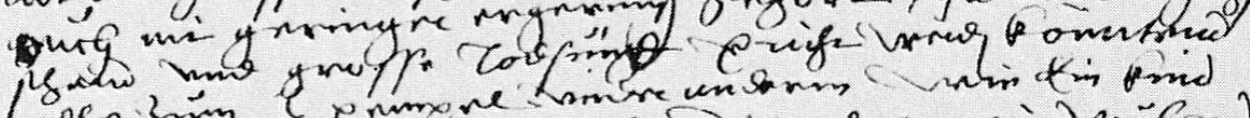

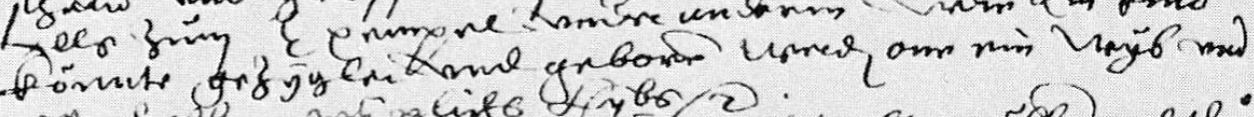

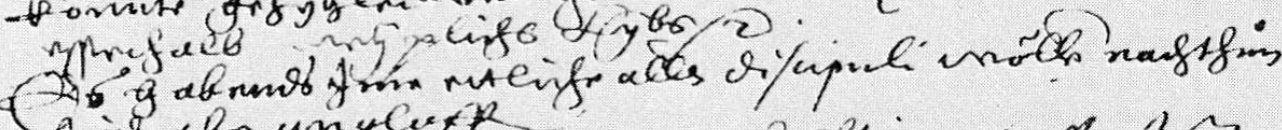

finis abre unylesth

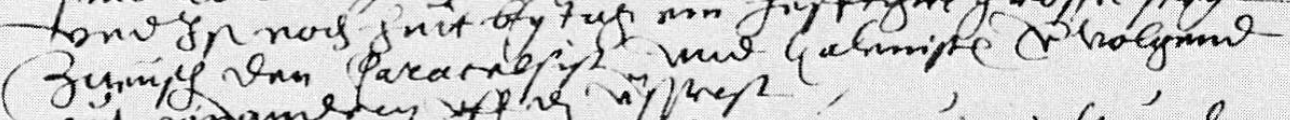

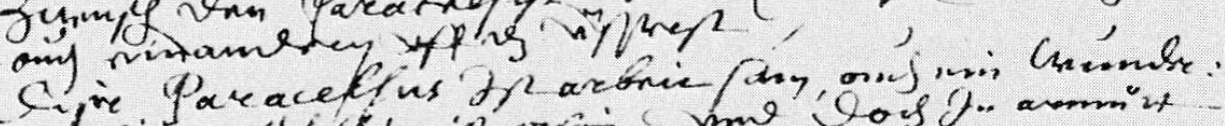

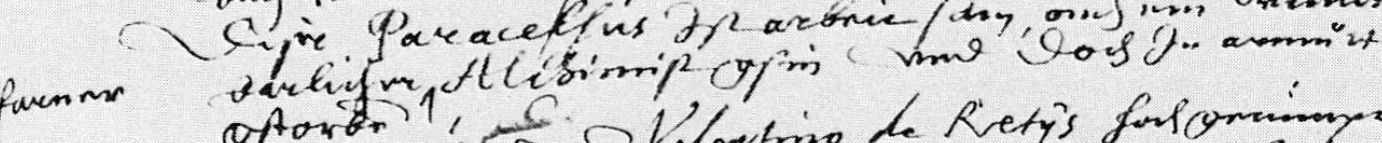

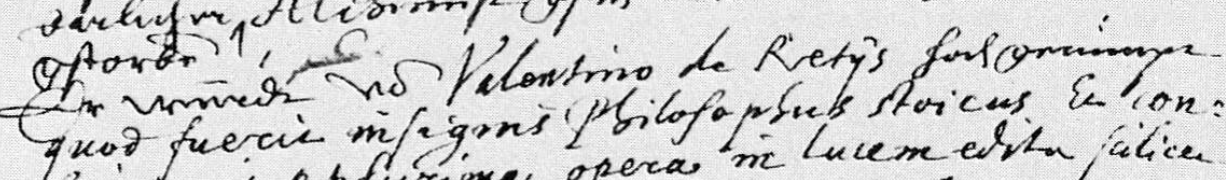

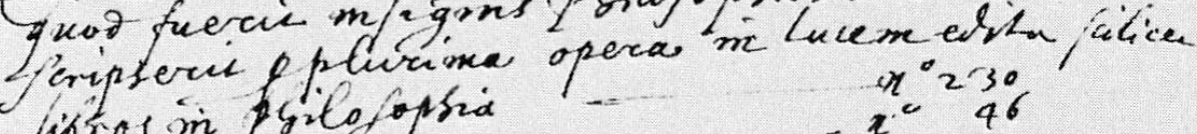
libros in pizito op

on secrian in oletferratica

- Je Sbeopiratha

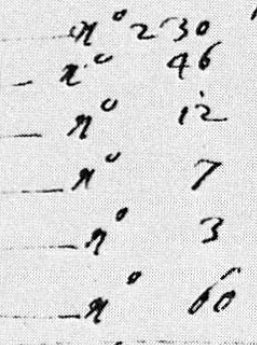

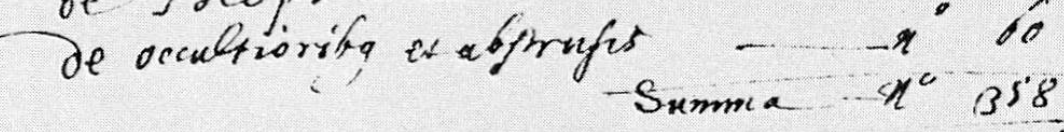

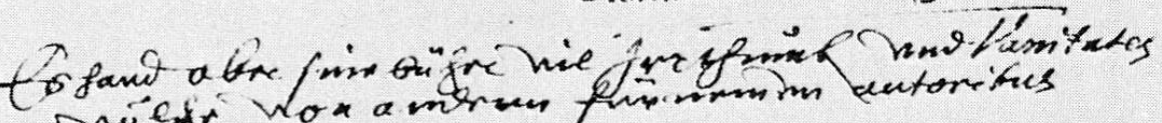

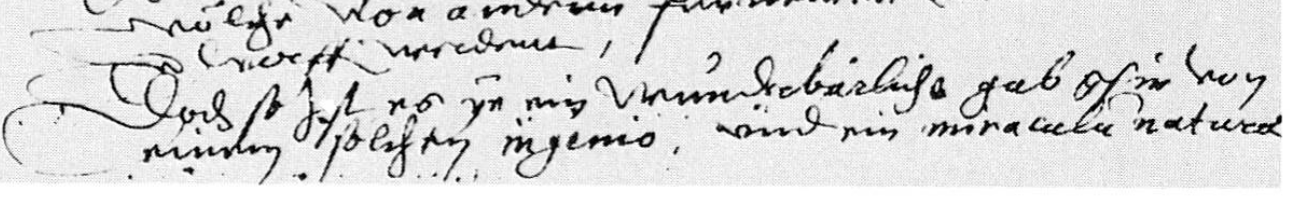


gelertern schriften gelesen het. derhalben lieber leser, nimm dises Theophrasti schriften in gutem und für lieb auf und scheue dich nicht, die selbigen höher zu achten dan der alten, ob sie schon neu zu sein vermeint werden. Vale.

Die Bücher-Spezifikation stimmt bis auf die Verteilung 46 (40) und 60 (66) genau überein.

Der Brief des Valentius de Retijs hat Adam von Bodenstein 1562 als Werbepamphlet für Paracelsus veröffentlichen lassen. Er sei jedoch, wie Sudhoff ${ }^{4}$ ausführt, als reklamehaftes Lobesepistel und Schelmenstück aus dem Kreise seiner Oberrheiner Anhänger der Jahre 1525/1527 zu betrachten, und es wäre verlorene Liebesmühe, hinter dem Namen ${ }^{5}$ eine historische Autorität suchen zu wollen.

Eines ist sicher: Cysat war kein Gegner des Paracelsus -, ihn aber als Paracelsist einzuordnen, geht entschieden zu weit. Er hat für den Hohenheimer eine gewisse Achtung, besaß von ihm einige Bücher, die er - wo es ihm paßte - für seine Arbeit benutzte.

\section{Literatur}

${ }^{1}$ Josef Schmid, Collectanea chronica und denkwürdige Sachen pro Chronica Lucernensi et Helvetiae. I. Band: Collectanea zur Geschichte der Stadt Luzern. Dritter Teil: Observationes variae tam jucundae quam utiles de rebus physicis, naturabilibus ac medicinalibus, Luzern 1972, S. $80 \mathrm{f}$.

${ }^{2}$ Josef Schmid, Collectanea ... Erste Abteilung, Stadt und Kanton Luzern. Erster Band. Erster Teil. Collectanea zur Geschichte der Stadt Luzern. Luzern 1969, S. XXII.

${ }^{3}$ Paracelsus. Theophrastus von Hohenheim genannt Paracelsus, Sämtliche Werke. 1. Abt. Medizinische, naturwissenschaftliche und philosophische Schriften, hrsg. von Karl Sudhoff, I-XIV, München usw. 1922-1933. Hier: III, 562.

${ }^{4}$ Paracelsus (Sudhoff) III, Vorwort, S. LII und LIII.

${ }^{5}$ Weitere Vorkommen in Handschriften, siehe: Karl Sudhoff, Versuch einer Kritik der Echtheit der Paracelsischen Schriften. II. Theil. Paracelsische Handschriften. 1. Hälfte. Berlin 1898, S. 93 (Valentius vom Riess), S.94 (Valentinus vom Riess), S.186 (Valentinus De Retijs); 2. Hälfte, Berlin 1899, S.434 (Valentinus deRetiis).

\section{Summary}

The Swiss Pharmacist and Historian Renward Cysat presents in his "Collectanea" an appreciation both of the activities and writings of Paracelsus. In addition, he mentions the quarrels between "Paracelsists" and "Galenists" without taking position himself.

Apoth. Dr.phil. Willem F.Daems, Postfach 40, CH-4144 Arlesheim 